\title{
Microvascular invasion of hepatocellular carcinoma predicts microvascular invasion of its recurrence: potential implications for salvage liver transplantation?
}

\author{
Stefan Heinrich ${ }^{1 \#}$, Jens Mittler ${ }^{1 \#}$, Juliane Theurer ${ }^{1}$, Dirk A. Ridder ${ }^{2}$, Jens U. Marquardt ${ }^{3}$, \\ Arndt Weinmann ${ }^{4}$, Uwe Scheuermann ${ }^{5}$, Gerd Otto ${ }^{1}$, Peter R. Galle ${ }^{4}$, Beate K. Straub ${ }^{2 *}$, Hauke Lang ${ }^{1 *}$ \\ ${ }^{1}$ General, Visceral and Transplantation Surgery, University Hospital of Mainz, Mainz, Germany; ${ }^{2}$ Institute of Pathology, University Hospital of \\ Mainz, Mainz, Germany; ${ }^{3}$ Department of Medicine I, University Medical Center Schleswig-Holstein, Campus Lübeck, Germany; ${ }^{4}$ Department of \\ Medicine I, University Hospital of Mainz, Mainz, Germany; ${ }^{5}$ Visceral, Transplantation, Thoracic and Vascular Surgery, University Hospital Leipzig, \\ Leipzig, Germany \\ Contributions: (I) Conception and design: S Heinrich, BK Straub, JU Marquardt; (II) Administrative support: G Otto, H Lang, PR Galle; (III) \\ Provision of study materials or patients: A Weinmann, BK Straub; (IV) Collection and assembly of data: J Theurer, U Scheuermann; (V) Data \\ analysis and interpretation: J Mittler, DA Ridder, BK Straub; (VI) Manuscript writing: All authors; (VII) Final approval of manuscript: All authors. \\ \#These authors contributed equally to this work. \\ *These authors contributed equally to this work. \\ Correspondence to: Stefan Heinrich, MD. General, Visceral and Transplantation Surgery, University Hospital of Mainz, Langenbeckstrasse 1, 55131 \\ Mainz, Germany. Email: stefan.heinrich@unimedizin-mainz.de.
}

Background: Microvascular invasion (MVI) can only be assessed on a full surgical specimen. We aimed at evaluating, whether the histology of the primary tumor is predictive of MVI in a hepatocellular carcinoma (HCC) recurrence.

Methods: Patients, who underwent liver resection or orthotopic liver transplantation (OLT) for recurrent HCC from January 2001 until June 2018 were eligible for this retrospective analysis. Resected specimens were evaluated for HCC subtype/morphology, vessels encapsulating tumor clusters (VETC)-pattern and MVI. Dichotomous parameters were analyzed using $\chi^{2}$-test and $\phi$-values, with $\mathrm{P}$ values $<0.05$ being considered significant.

Results: Of 230 HCC recurrences, 37 (16.1\%) underwent repeated liver resection ( $\mathrm{n}=22)$ or OLT ( $\mathrm{n}=15)$. Of these, $67.6 \%$ initially exceeded the Milan criteria. MVI correlated Milan criteria $(\mathrm{P}=0.005)$, tumor size $(\mathrm{P}=0.015)$ and VETC-pattern $(\mathrm{P}=0.034)$ in the primary specimen. The recurrences shared many features of the primary HCC such as tumor grade $(\mathrm{P}=0.002)$, VETC-pattern $(\mathrm{P}=0.035)$, and MVI $(\mathrm{P}=0.046)$. In recurrences, however, only the concordance with the Milan criteria correlated with MVI $(\mathrm{P}=0.018)$. No patient without MVI in the primary HCC revealed MVI on early recurrence ( $<2$ years) $(\mathrm{P}=0.035)$.

Conclusions: HCC recurrences share many biological features of the primary tumor. Moreover, early recurrences of MVI-negative HCC never revealed MVI. This finding offers novel concepts, e.g., patient selection for salvage OLT.

Keywords: Microvascular invasion (MVI); resection selection; vessels encapsulating tumor clusters (VETC); angioinvasion; salvage transplantation

Submitted Aug 23, 2021. Accepted for publication Nov 18, 2021. Published online Mar 03, 2022.

doi: $10.21037 / \mathrm{hbsn}-21-346$

View this article at: https://dx.doi.org/10.21037/hbsn-21-346 


\section{Introduction}

The optimal management of hepatocellular carcinoma (HCC) is determined by the extent and pattern of the tumor as well as liver function due to the predominant association of HCC with chronic liver diseases such as fibrosis, steatohepatitis or cirrhosis (1). Thus, orthotopic liver transplantation (OLT) is considered the optimal treatment for HCC patients with liver cirrhosis, since recurrencefree survival is unaffected if patients are transplanted within the Milan criteria (2). Moreover, experience of large international centers also demonstrates excellent long-term prognosis for selected patients with HCC beyond the Milan criteria (3). Consequently, an extension of the transplant criteria has been advocated by an HCC consensus conference in 2010 (4).

Extending the indications for OLT in HCC could potentially cure some patients, but would also further encumber the limited donor pool. Therefore, patients with compensated liver cirrhosis (Child A) may be offered liver resection (LR) and (salvage) OLT as a rescue option in cases of tumor recurrence. This concept appears particularly attractive, since patients with a beneficial course after a curative LR may be spared from unnecessary OLT, and organs would be saved for the donor pool (5). On the other hand, a large proportion of initially transplantable patients drop-out during the follow-up period prior to a salvageOLT due to the development of contraindications for OLT (e.g., advanced age, comorbidities and extent of disease). Therefore, primary OLT revealed a better long-term outcome on an intention-to-treat basis than the salvage concept $(6,7)$.

While tumor size and number (included in the Milan criteria) are prognostic factors in HCC, the most important prognostic parameter for HCC recurrence remains microvascular invasion (MVI) (7-12). Ideally, patient selection for OLT would be based on biological criteria such as the MVI-status rather than clinical surrogate parameters. However, the assessment of, e.g., the MVIstatus of an HCC requires the full histological work-up of the HCC $(9,10):$ MVI characterizes the vascular invasion of HCC around rather than inside the HCC. In contrast to this characteristic peritumoral feature, a particular intratumoral vessels encapsulating tumor clusters (VETC)pattern has been proposed as additional prognostic feature, which correlates with early metastasis in HCC (13).

Currently, it is unknown whether a recurrent HCC shares the biological features of its primary tumor.
Moreover, the significance of VETC-pattern in this context is unknown. Therefore, we tested, in a highly selected cohort of patients, whether the presence or absence of MVI and VETC-pattern in the primary HCC predicted their occurrence in the HCC recurrence in patients amenable to surgical therapy. By this, we wanted to assess whether a primary LR could help to select patients with HCC outside the Milan criteria for OLT in this setting. We present the following article in accordance with the STROBE reporting checklist (available at https://hbsn.amegroups.com/article/ view/10.21037/hbsn-21-346/rc).

\section{Methods}

Patients who underwent a second LR or liver transplantation for recurrent HCC at our center between January 2001 and June 2018 were included into this analysis. Biopsies-only for the primary diagnosis as well as the confirmation of recurrence were excluded from this analysis. Also, tumors with macroscopic vascular infiltration were ineligible.

All clinical parameters were extracted from a prospective clinical database and analyzed retrospectively. Due to the long study period and the limited availability of computed tomography images of patients operated more than 5 years ago at our center, tumor extent in the primary specimen and tumor recurrence were assessed histologically.

The study was performed according to the ethical guidelines of the Declaration of Helsinki (as revised in 2013) and was approved by the institution's human research committee (2018-13857_1).

\section{Histological assessment}

Routinely, one section per $1 \mathrm{~cm}$ HCC-diameter was assessed and at least one representative of the tumor border. All primary and recurrent HCC were reassessed by two pathologists (BK Straub, DA Ridder) for histological subtype, micro-/macrovascular tumor infiltration, as well as routine CD34-immunohistochemistry and grading on hematoxylin \& eosin (H\&E) slides.

MVI was defined as nests of tumor cells in the vascular cavity of small arteries or veins, that were not grossly recognizable and could by identified only by conventional light microscopic observation or immunohistochemical techniques. Immunohistochemistry for CD34 was performed in a representative tumor section at the border to non-neoplastic liver parenchyma, if peritumoral tumor 


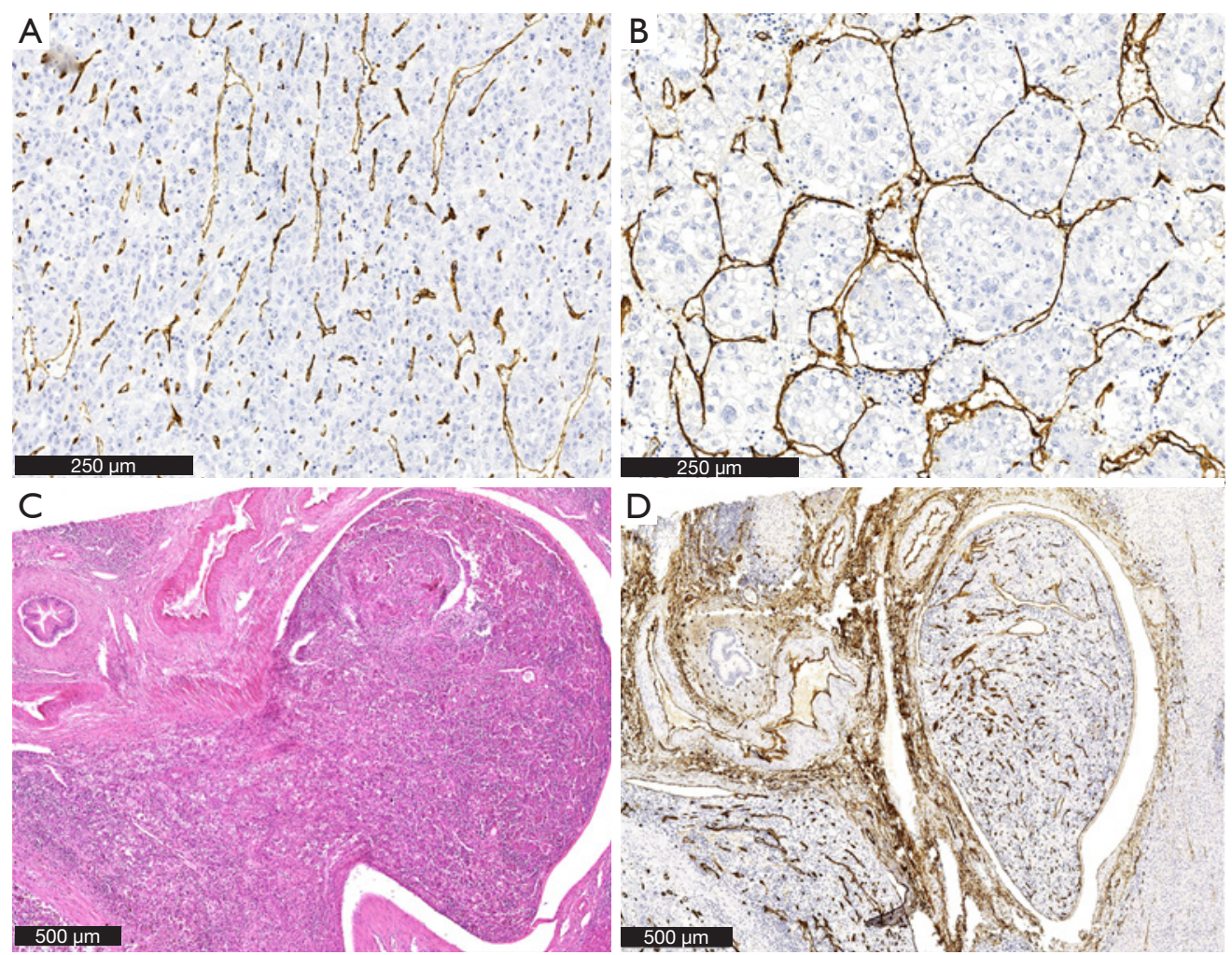

Figure 1 Histological scoring of VETC-patterns and microvascular invasion. CD34-staining demonstrates vessels in HCC without lining of tumor cell clusters (A). In contrast, VETC are nicely delineated in (B) (IHC staining). A microvascular invasion is displayed on H\&E (C) and CD34 (D) staining. VETC, vessels encapsulating tumor clusters; HCC, hepatocellular carcinoma; IHC, immunohistochemistry; H\&E, hematoxylin \& eosin.

deposits were detected and the relation to a vessel remained unclear on H\&E staining. Slides were graded MVI-positive or MVI-negative according to the H\&E + CD34 staining (Figure 1).

In addition, the morphology of the primary and recurrent tumors was assessed: a scoring in "similar" or "different" type of differentiation was provided. The HCC subtype was assessed according to the WHO classification of 2019 (e.g., fibrolamellar, steatohepatitic, clear-cell, macrotrabecular, chromophobe, scirrhous, lymphocyte-rich, neutrophil-rich, etc.) and in case, the HCC was of no special subtype, the predominant differentiation (e.g., steatotic, solid, trabecular, pseudo-glandular) was described (14).

Moreover, the VETC-pattern of each primary and recurrent HCC was characterized with routine CD34 immunohistochemistry. The slides were graded as VETCpositive and VETC-negative according to Fang et al. (13). In intermediate cases with unclear or only focal and not representative vessels encapsulating pattern in the whole tumor, the tumors were attributed VETC-negative (Figure 1).
If more than one HCC nodule was present, H\&Estained slides of all nodules were analyzed for MVI. Usually, the largest lesion was stained for CD34. However, when MVI was suspected, but could not be confirmed with standard light microscopy, the respective paraffin block was additionally stained with antibodies against CD34. VETC pattern was analyzed on all samples stained for CD34.

\section{Statistical analysis}

All values are presented as median (range). Proportions were calculated and compared by standard 2-by-2 tables using the $\chi^{2}$ test to test whether categorical data of two groups differed. Correlations between dichotomous variables were tested in 2-by-2 tables using the $\phi$-value. Continuous parameters were compared by the Mann-Whitney $U$ test. Survival analyses were performed according to the Kaplan-Meier method using the Log-rank test. P values $<0.05$ were considered significant. All statistical analyses were performed using SPSS software (version 11.5, SPSS, 
Table 1 Patient/tumor characteristics

\begin{tabular}{|c|c|c|}
\hline Characteristics & Primary HCC & $\begin{array}{l}\mathrm{HCC} \\
\text { recurrence }\end{array}$ \\
\hline Sex, female/male & \multicolumn{2}{|c|}{$7 / 30$} \\
\hline Age, median [range], years & \multicolumn{2}{|c|}{$61[32-81]$} \\
\hline Liver cirrhosis & \multicolumn{2}{|c|}{$24 / 37$ (64.9\%) } \\
\hline Child B/C & \multicolumn{2}{|c|}{-} \\
\hline Diabetes & \multicolumn{2}{|c|}{$8 / 37$ (21.6\%) } \\
\hline BMI, median [range], $\mathrm{kg} / \mathrm{m}^{2}$ & \multicolumn{2}{|c|}{$25.4[18.6-33.7]$} \\
\hline$<20$ & \multicolumn{2}{|c|}{$3(8.1 \%)$} \\
\hline $20-25$ & \multicolumn{2}{|c|}{$12(32.4 \%)$} \\
\hline$>25, \leq 30$ & \multicolumn{2}{|c|}{$8(21.6 \%)$} \\
\hline$>30$ & \multicolumn{2}{|c|}{$7(18.9 \%)$} \\
\hline \multicolumn{3}{|l|}{ Etiology } \\
\hline Hepatitis B & \multicolumn{2}{|c|}{7 (18.9\%) } \\
\hline Hepatitis C & \multicolumn{2}{|c|}{$8(21.6 \%)$} \\
\hline Alcohol & \multicolumn{2}{|c|}{9 (24.3\%) } \\
\hline Non-alcoholic steatohepatitis & \multicolumn{2}{|c|}{$2(5.4 \%)$} \\
\hline Other & \multicolumn{2}{|c|}{$2(5.4 \%)$} \\
\hline Unknown & \multicolumn{2}{|c|}{$9(24.3 \%)$} \\
\hline \multicolumn{3}{|l|}{ Surgery } \\
\hline Segment resection (lap/open) & 19 & 22 \\
\hline Left-lateral resection (lap/open) & 4 & - \\
\hline Mesohepatectomy & 2 & - \\
\hline hemihepatectomy & 10 & - \\
\hline Extended hemihepatectomy & 2 & - \\
\hline OLT & - & 15 \\
\hline \multirow[t]{2}{*}{ Bridging before OLT } & - & TACE $n=5$ \\
\hline & - & $\begin{array}{c}\text { Additional: } \\
\text { IRE } n=1 ; \text { RFA } \\
n=1\end{array}$ \\
\hline Ro & $34(89.5 \%)^{*}$ & $35(92.1 \%)$ \\
\hline \multicolumn{3}{|l|}{ Grading } \\
\hline 1 & 7 & 5 \\
\hline 2 & 25 & 23 \\
\hline 3 & 4 & 8 \\
\hline 4 & 1 & - \\
\hline None & - & $1^{\star \star}$ \\
\hline
\end{tabular}

Table 1 (continued)
Table 1 (continued)

\begin{tabular}{|c|c|c|}
\hline Characteristics & Primary HCC & $\begin{array}{l}\mathrm{HCC} \\
\text { recurrence }\end{array}$ \\
\hline Within Milan criteria & $12(32.4 \%)$ & $24(64.9 \%)$ \\
\hline $\begin{array}{l}\text { Max. diameter of HCC, median } \\
\text { [range], } \mathrm{cm}\end{array}$ & $6.5[4.0-26.0]$ & $2.1[0.8-7.0]$ \\
\hline Number of $\mathrm{HCC}$, median [range] & $1[1-3]$ & $1[1-6]$ \\
\hline Microvascular invasion & $18(48.6 \%)$ & $11(29.7 \%)$ \\
\hline VETC $^{+}$ & $12(32.4 \%)$ & $10(27.0 \%)$ \\
\hline \multicolumn{3}{|l|}{ HCC subtype } \\
\hline Trabecular & $32(86.5 \%)$ & $31(83.8 \%)$ \\
\hline Clear cell & $1(2.7 \%)$ & $2(5.4 \%)^{\star \star \star}$ \\
\hline Steatohepatitic & $1(2.7 \%)$ & $1(2.7 \%)$ \\
\hline Macrotrabecular & $1(2.7 \%)$ & $1(2.7 \%)$ \\
\hline Fibrolamellar & $1(2.7 \%)$ & $1(2.7 \%)$ \\
\hline Mixed HCC/CCC & $1(2.7 \%)$ & $1(2.7 \%)$ \\
\hline \multicolumn{3}{|c|}{$\begin{array}{l}{ }^{*} \text {, one only written histological report available; }{ }^{* *} \text {, no grading } \\
\text { available after TACE; }{ }^{* \star} \text {, initially trabecular with partial clear cell type, } \\
\text { recurrence with clear cell type. HCC, hepatocellular carcinoma, } \\
\text { BMI, body mass index; CCC, cholangiocarcinoma; OLT, orthotopic } \\
\text { liver transplantation; TACE, transarterial chemoembolization; IRE, } \\
\text { irreversible electroporation; RFA, radiofrequency ablation; VETC } \\
\text { vessels encapsulating tumor clusters. }\end{array}$} \\
\hline
\end{tabular}

Chicago, IL, USA).

\section{Results}

During the study period, 428 patients underwent LR for HCC, and 230 patients have developed a documented recurrence. Of these, 37 patients $(16.1 \%)$ underwent repeated LR $(n=22)$ or liver transplantation $(n=15)$ for curative treatment of HCC recurrence. One patient had undergone primary HCC resection at another hospital and the original specimen was not available for histological reassessment. Only clinical parameters were used from this patient. The median period between the first LR and re-resection or liver transplantation was 19.1 months (5.1-95.0 months).

Tumor parameters of the primary and recurrent HCC are listed in Table 1. Most of the recurrent HCC (34/37; 91.9\%) showed a conventional histological morphology similar to the primary tumor. Also, the histological subtype of the primary and recurrent HCC was congruent in all 
patients except one. This patient developed recurrence more than 2 years after the primary tumor in hepatitis $\mathrm{B}$ cirrhosis: while the primary HCC revealed features of a trabecular and clear cell type, the recurrent tumor was classified as clear cell type, only.

\section{Primary tumor characteristics}

Most patients (67.6\%) presented with an HCC outside the Milan criteria at study entry, and $67 \%$ of these HCC revealed MVI. Concordance with the Milan criteria $(\phi=0.471$; $\mathrm{P}=0.005)$ and the size of the tumors $(\phi=0.405 ; \mathrm{P}=0.015)$ correlated well with the presence of MVI: 10/13 (76.9\%) HCC below $5 \mathrm{~cm}$ revealed no MVI, and 15/24 (62.5\%) HCC above $5 \mathrm{~cm}$ exhibited MVI. On the other hand, three HCC within the Milan criteria revealed MVI, and 9 HCC above $5 \mathrm{~cm}$ did not contain MVI ( $\mathrm{P}=0.035)$. The presence of MVI correlated with VETC-positive pattern $(\phi=0.354 ; \mathrm{P}=0.034)$ in contrast to the tumor grading $(\phi=0.473 ; \mathrm{P}=0.41)$.

The median recurrence-free survival after primary surgery was shorter for HCC with MVI compared to no MVI (550 vs. 637 days, $\mathrm{P}=0.442$ ), $\mathrm{VETC}^{+}$compared to $\mathrm{VETC}^{-}$pattern $(383$ vs. 572 days, $\mathrm{P}=0.635)$ and concordance compared to disagreement with Milan criteria (414 vs. 567 days, $\mathrm{P}=0.617$ ). However, these differences did not reach statistical significance.

\section{Recurrent tumor characteristics}

Among the HCC recurrences, neither VETC-pattern ( $\phi$ $=-0.127 ; \mathrm{P}=0.446)$, tumor grade $(\phi=0.287 ; \mathrm{P}=0.384)$, nor size of the HCC ( $\phi=0.043 ; \mathrm{P}=0.798)$ correlated with MVI in the HCC recurrence. Only the concordance with the Milan criteria revealed a significant correlation with an MVI in the HCC recurrence $(\phi=0.388 ; \mathrm{P}=0.018)$. Again, four recurrent HCC $(16.7 \%)$ revealed MVI despite being within the Milan criteria, and 6 out of 13 (46.2\%) recurrences outside Milan criteria did not contain MVI.

The concordance with the Milan criteria of the primary HCC was not associated with the Milan-concordance of the recurrence $(\phi=0.250 ; \mathrm{P}=0.134)$. The tumor grade $(\phi=0.849$; $\mathrm{P}=0.002)$, the VETC-pattern $(\phi=0.351 ; \mathrm{P}=0.035)$ and MVI $(\phi=0.324 ; \mathrm{P}=0.046)$ of the primary and recurrent HCC correlated significantly.

\section{Prediction of MVI in recurrent $H C C$}

MVI was detected in 18 primary and in 11 recurrent HCC.
While 8 patients with MVI in the primary HCC also had a MVI in the secondary HCC, only three patients without MVI in the primary HCC revealed a vascular infiltration in the recurrent $\mathrm{HCC}\left(\phi=0.263 ; \mathrm{P}=0.106 ; \chi^{2} \mathrm{P}=0.164\right)$. None of the above-mentioned histological parameters of the primary HCC was associated with MVI of the recurrent HCC.

\section{Subgroup analysis}

Since recurrences within two years are considered true recurrences of the primary HCC $(1,12)$, we performed a subgroup analysis of these early recurrences: 22 patients (59.5\%) developed the recurrence within 2 years (Table 2). Of these, 11 patients had MVI in the primary HCC, and 6 of these 11 patients (54.5\%) also revealed MVI in the recurrence. Most importantly, recurrent HCC of MVInegative primary tumors never had MVI $(\mathrm{P}=0.005)$, which translates to a negative predictive factor of $100 \%$ and a positive predictive factor of $54.5 \%$ (Figure 2).

In this subgroup, only eight primary HCC (33.3\%) were within the Milan-criteria. Neither concordance with the Milan criteria $(\mathrm{P}=0.189)$, number $(\mathrm{P}=1.0)$, size $(\mathrm{P}=0.098)$, tumor grading $(\mathrm{P}=0.140)$ nor VETC-pattern $(\mathrm{P}=0.065)$ of the primary HCC predicted MVI of the recurrence.

\section{Discussion}

Currently, the best treatment for patients with HCC in cirrhosis is OLT (1). Although still the standard for patient selection in many regions, most experts agree that selected patients with HCC exceeding the Milan criteria benefit as much from OLT as those within the Milan criteria $(2,4)$. One reason for this discrepancy is that MVI is a much stronger predictor of the tumor aggressiveness than the tumor size or numbers $(8,9)$. However, various attempts have failed to predict MVI in the HCC, and just recently, several prognostic models including various clinical, histological as well as biological parameters have been proposed in order to estimate the risk of MVI $(9,15-19)$.

In this analysis, we found that recurrent HCC share many of the biological features of the primary HCC. Most importantly, early recurrences ( $<2$ years) of an MVInegative HCC were always MVI-negative. Moreover, only about half of recurrent HCC from MVI-positive tumors revealed MVI.

In accordance with the literature, HCC within the Milan criteria were significantly more often MVI-negative in the primary specimen in our series, and a fair number of HCC 
Table 2 Patient/tumor characteristics of early recurrences

\begin{tabular}{|c|c|c|}
\hline Characteristics & Primary HCC & HCC recurrence \\
\hline Sex, female/male & \multicolumn{2}{|c|}{$4 / 18$} \\
\hline $\begin{array}{l}\text { Age, median [range], } \\
\text { years }\end{array}$ & \multicolumn{2}{|c|}{64 [32-79] } \\
\hline Liver cirrhosis & \multicolumn{2}{|c|}{$14 / 22(63.6 \%)$} \\
\hline Ro & $20(90.1 \%)$ & $21(95.5 \%)$ \\
\hline \multicolumn{3}{|l|}{ Grading } \\
\hline 1 & 4 & 2 \\
\hline 2 & 15 & 16 \\
\hline 3 & 2 & 3 \\
\hline 4 & 1 & - \\
\hline None & - & $1^{*}$ \\
\hline VETC & 7 (31.8\%) & $9(40.9 \%)$ \\
\hline Within Milan criteria & 8 (36.4\%) & $16(72.7 \%)$ \\
\hline $\begin{array}{l}\text { Max. diameter of HCC, } \\
\text { median [range], } \mathrm{cm}\end{array}$ & $6.5[4.0-26.0]$ & $2.2[0.8-7.0]$ \\
\hline $\begin{array}{l}\text { Number of HCC, median } \\
\text { [range] }\end{array}$ & $1[1-2]$ & $1[1-5]$ \\
\hline Microvascular invasion & $11(50.0 \%)$ & $6(27.3 \%)$ \\
\hline \multicolumn{3}{|l|}{ HCC subtype } \\
\hline Trabecular & $19(86.4 \%)$ & $19(86.4 \%)$ \\
\hline Clear cell & $1(4.5 \%)$ & $1(4.5 \%)$ \\
\hline Steatohepatitic & 0 & 0 \\
\hline Macrotrabecular & $1(4.5 \%)$ & $1(4.5 \%)$ \\
\hline Fibrolamellar & 0 & 0 \\
\hline Mixed HCC/CCC & $1(4.5 \%)$ & $1(4.5 \%)$ \\
\hline
\end{tabular}

*, no grading available after TACE. CCC, cholangiocarcinoma; $\mathrm{HCC}$, hepatocellular carcinoma; VETC, vessels encapsulating tumor clusters; TACE, transarterial chemoembolization.

exceeded the Milan criteria but did not reveal MVI $(8,20)$. These patients are currently excluded from OLT based on imaging criteria, only, despite their favorable prognosis according to their biological parameters.

The current concept in the literature distinguishes early ( $<2$ years) from late $(>2$ years) recurrences $(1,12,21)$ : recurrences within 2 years are considered true recurrences (metastases) while recurrences thereafter are considered de novo HCC. The latter are supposed to develop independently from a previous HCC and are mainly triggered by the underlying liver disease. However, recurrences within 2 years from surgery may also be de novo HCC due to the same stimulus in the diseased liver, which would also explain the lower HCC recurrence rates of patients with sustained virological response of hepatitis C compared to non-responders (22). Thus, we separately analyzed patients according to the timing of their tumor recurrences: our results are in line with the literature, which suggests a very similar tumor biology particularly in the subgroup of patients with early recurrence compared to the initial HCC, supporting the theory of true recurrences rather than de novo HCC. Irrespective of the recurrencefree period and the occurrence of MVI in either surgical specimen, HCC morphology was comparable and HCC subtype was congruent in the vast majority of patients between the primary and recurrent HCC.

Our patient cohort has a predominance of viral hepatitis as underlying liver disease. According to the general population in the area of our center, a large proportion of patients also had alcoholic liver disease. Considering the high number of recurrences ( $>50 \%$ ) after an apparently curative LR, only $16 \%$ were amenable to a surgical therapy again and were included into this analysis. Congruent figures have been reported from Asia with a $17 \%$ rate of local therapies for HCC recurrences (23). Expectedly, these recurrences were smaller and (therefore) more likely to meet the Milan criteria. Similarly, the Milan criteria of the primary and recurrent HCC did not match in our cohort despite concordance of many histological and biological factors. This finding is presumably due to the successful surveillance of patients after surgery for HCC, and some of the recurrences have been detected early and might have exhibited MVI with increasing size.

Although the Milan criteria were originally defined from histological specimen, they are nowadays applied by imaging analysis in the clinical routine (2). However, a recent analysis demonstrated that the extent of many HCC is still underestimated and that the prognosis is related to the histological extent of the disease (24). In this analysis, we also defined the size and distribution of the HCC on the histological specimen, since imaging quality varied over time and original images of several patients were unavailable due to the long study period in some patients.

The VETC-pattern also revealed a significant concordance in the primary and recurrent HCC in our series, which adds further evidence that recurrences harbor similar biological features to the primary HCC. This histological feature also defines an aggressive subgroup of 


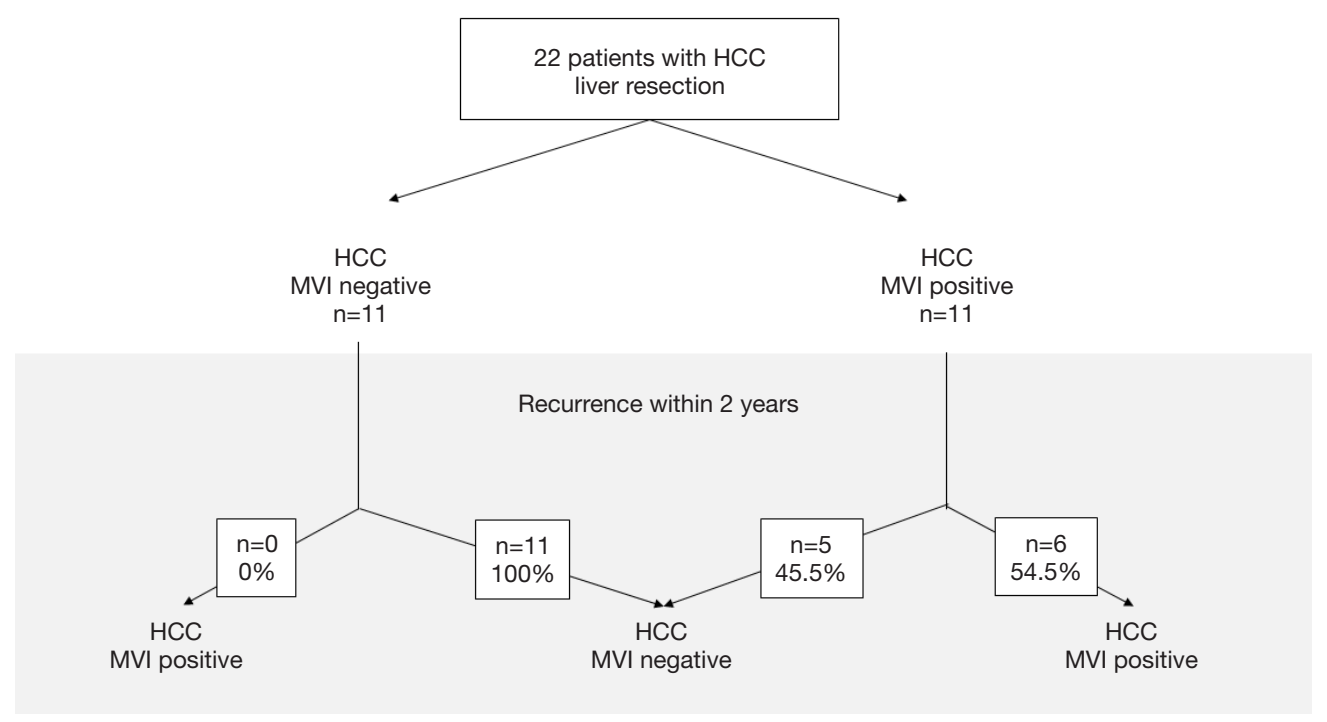

Figure 2 MVI in the primary HCC predicts MVI in the early recurrent HCC. The flow diagram demonstrates the distribution of MVI in the recurrent HCC in dependence of the MVI in the primary specimen. HCC, hepatocellular carcinoma; MVI, microvascular invasion.

HCC and may be even more relevant than MVI $(13,25)$. The VETC-pattern correlated well with the MVI status of the primary but not the recurrent HCC in our series. However, since only the MVI of the primary HCC significantly predicted the MVI of early recurrences, MVI still appears to be a stronger prognostic factor than VETC in this particular clinical scenario. Since VETC on the other hand may be assessed on tumor biopsies, this parameter remains a promising prognostic factor and requires further evaluation.

Due to the estimated time on the waiting list and organ shortage in many regions, staged concepts of surgery prior to OLT have been proposed in compensated cirrhosis in order to avoid unnecessary OLT and save organs. In this context, surgery may serve as "bridge to transplantation" in two ways: "salvage OLT" may rescue recurrent HCC or LR may be used as "resection selection" concept. In the latter concept, OLT would be performed after resection of the HCC but prior to tumor recurrence based on the biological criteria of the pathological evaluation of a complete surgical specimen (26). In a "resection selection" concept, HCC outside the Milan-criteria might be offered OLT prior to recurrence in the absence of MVI due to the beneficial prognosis. OLT may even be beneficial for early recurrences, if the primary HCC did not reveal MVI irrespective of its Milan criteria based on our results, since such a recurrence is very unlikely to contain MVI (26). In reverse, patients with MVI-positive HCC would be excluded from a "resection selection" concept irrespective of the tumor extent due to the estimated recurrence rate (8). However, nearly half of the patients with MVI in the primary HCC did not show MVI in the recurrence in our series. Similarly, about $50 \%$ of patients with singular MVI-positive HCC $(<5 \mathrm{~cm})$, who were listed for OLT after resection remained recurrence-free during the observation period in a series of the Barcelona group (27). Although this recurrence rate is higher than for patients inside the Milan criteria (2), a categorical exclusion of MVI-positive HCC from salvage transplantation concepts is not justified. Further parameters are required to better select patients for the respective treatment concepts always considering the general organ shortage.

Due to the limited sample size of a highly selective cohort of patients treated at a single center for HCC, results of this analysis should be considered hypothesis generating rather than as a proof of principle. These data should trigger further research including a prospective validation in larger (multicenter) sets of patients.

In conclusion, this analysis demonstrates that although recurrences of HCC after LR share many biological characteristics of their primary HCC early tumor recurrences (<2 years) of MVI-negative HCC never revealed MVI.

\section{Acknowledgments}

Funding: None. 


\section{Footnote}

Reporting Checklist: The authors have completed the STROBE reporting checklist. Available at https://hbsn. amegroups.com/article/view/10.21037/hbsn-21-346/rc

Data Sharing Statement: Available at https://hbsn. amegroups.com/article/view/10.21037/hbsn-21-346/dss

Conflicts of Interest: All authors have completed the ICMJE uniform disclosure form (available at https://hbsn. amegroups.com/article/view/10.21037/hbsn-21-346/ coif). HL serves as an unpaid editorial board member of Hepatobiliary Surgery and Nutrition. JUM participated in advisory boards for Bayer, Eisai, AstraZeneca, Roche. PRG receives consulting fees from Bayer, Boston Scientific, AstraZeneca, Adaptimmune, BMS, Eisai, MSD, Sirtex, Lilly, Roche, Guerbet, Ipsen; and payment or honoraria from Bayer Adaptimmune, Sirtex, Lilly, Roche, Ipsen. The other authors have no conflicts of interest to declare.

Ethical Statement: The authors are accountable for all aspects of the work in ensuring that questions related to the accuracy or integrity of any part of the work are appropriately investigated and resolved. The study was performed according to the ethical guidelines of the Declaration of Helsinki (as revised in 2013) and was approved by the institution's human research committee (2018-13857_1).

Open Access Statement: This is an Open Access article distributed in accordance with the Creative Commons Attribution-NonCommercial-NoDerivs 4.0 International License (CC BY-NC-ND 4.0), which permits the noncommercial replication and distribution of the article with the strict proviso that no changes or edits are made and the original work is properly cited (including links to both the formal publication through the relevant DOI and the license). See: https://creativecommons.org/licenses/by-nc-nd/4.0/.

\section{References}

1. European Association for the Study of the Liver. Electronic address: easloffice@easloffice.eu; European Association for the Study of the Liver. EASL Clinical Practice Guidelines: Management of hepatocellular carcinoma. J Hepatol 2018;69:182-236.

2. Mazzaferro V, Regalia E, Doci R, et al. Liver transplantation for the treatment of small hepatocellular carcinomas in patients with cirrhosis. N Engl J Med 1996;334:693-9.

3. Mazzaferro V. Results of liver transplantation: with or without Milan criteria? Liver Transpl 2007;13:S44-7.

4. Clavien PA, Lesurtel M, Bossuyt PM, et al. Recommendations for liver transplantation for hepatocellular carcinoma: an international consensus conference report. Lancet Oncol 2012;13:e11-22.

5. Cherqui D, Laurent A, Mocellin N, et al. Liver resection for transplantable hepatocellular carcinoma: long-term survival and role of secondary liver transplantation. Ann Surg 2009;250:738-46.

6. Menahem B, Lubrano J, Duvoux C, et al. Liver transplantation versus liver resection for hepatocellular carcinoma in intention to treat: An attempt to perform an ideal meta-analysis. Liver Transpl 2017;23:836-44.

7. Bhangui P, Allard MA, Vibert E, et al. Salvage Versus Primary Liver Transplantation for Early Hepatocellular Carcinoma: Do Both Strategies Yield Similar Outcomes? Ann Surg 2016;264:155-63.

8. Mazzaferro V, Llovet JM, Miceli R, et al. Predicting survival after liver transplantation in patients with hepatocellular carcinoma beyond the Milan criteria: a retrospective, exploratory analysis. Lancet Oncol 2009;10:35-43.

9. Erstad DJ, Tanabe KK. Prognostic and Therapeutic Implications of Microvascular Invasion in Hepatocellular Carcinoma. Ann Surg Oncol 2019;26:1474-93.

10. Rodríguez-Perálvarez M, Luong TV, Andreana L, et al. A systematic review of microvascular invasion in hepatocellular carcinoma: diagnostic and prognostic variability. Ann Surg Oncol 2013;20:325-39.

11. Pommergaard HC, Rostved AA, Adam R, et al. Vascular invasion and survival after liver transplantation for hepatocellular carcinoma: a study from the European Liver Transplant Registry. HPB (Oxford) 2018;20:768-75.

12. Imamura $\mathrm{H}$, Matsuyama $\mathrm{Y}$, Tanaka $\mathrm{E}$, et al. Risk factors contributing to early and late phase intrahepatic recurrence of hepatocellular carcinoma after hepatectomy. J Hepatol 2003;38:200-7.

13. Fang JH, Zhou HC, Zhang C, et al. A novel vascular pattern promotes metastasis of hepatocellular carcinoma in an epithelial-mesenchymal transition-independent manner. Hepatology 2015;62:452-65.

14. Calderaro J, Ziol M, Paradis V, et al. Molecular and histological correlations in liver cancer. J Hepatol 2019;71:616-30.

15. Fuks D, Cauchy F, Fusco G, et al. Preoperative tumour 
biopsy does not affect the oncologic course of patients with transplantable HCC. J Hepatol 2014;61:589-93.

16. Nitta H, Allard MA, Sebagh M, et al. Prognostic Value and Prediction of Extratumoral Microvascular Invasion for Hepatocellular Carcinoma. Ann Surg Oncol 2019;26:2568-76.

17. Lee S, Kang TW, Song KD, et al. Effect of Microvascular Invasion Risk on Early Recurrence of Hepatocellular Carcinoma After Surgery and Radiofrequency Ablation. Ann Surg 2021;273:564-71.

18. Lei Z, Li J, Wu D, et al. Nomogram for Preoperative Estimation of Microvascular Invasion Risk in Hepatitis B Virus-Related Hepatocellular Carcinoma Within the Milan Criteria. JAMA Surg 2016;151:356-63.

19. Zhang X, Li J, Shen F, et al. Significance of presence of microvascular invasion in specimens obtained after surgical treatment of hepatocellular carcinoma. J Gastroenterol Hepatol 2018;33:347-54.

20. Pawlik TM, Delman KA, Vauthey JN, et al. Tumor size predicts vascular invasion and histologic grade: Implications for selection of surgical treatment for hepatocellular carcinoma. Liver Transpl 2005;11:1086-92.

21. Llovet JM, Di Bisceglie AM, Bruix J, et al. Design and endpoints of clinical trials in hepatocellular carcinoma. J Natl Cancer Inst 2008;100:698-711.

Cite this article as: Heinrich S, Mittler J, Theurer J, Ridder DA, Marquardt JU, Weinmann A, Scheuermann U, Otto G, Galle PR, Straub BK, Lang H. Microvascular invasion of hepatocellular carcinoma predicts microvascular invasion of its recurrence: potential implications for salvage liver transplantation? HepatoBiliary Surg Nutr 2023;12(2):183-191. doi: 10.21037/hbsn-21-346
22. Manthravadi S, Paleti S, Pandya P. Impact of sustained viral response postcurative therapy of hepatitis C-related hepatocellular carcinoma: a systematic review and metaanalysis. Int J Cancer 2017;140:1042-9.

23. Chan AC, Poon RT, Cheung TT, et al. Survival analysis of re-resection versus radiofrequency ablation for intrahepatic recurrence after hepatectomy for hepatocellular carcinoma. World J Surg 2012;36:151-6.

24. Ecker BL, Hoteit MA, Forde KA, et al. Patterns of Discordance Between Pretransplant Imaging Stage of Hepatocellular Carcinoma and Posttransplant Pathologic Stage: A Contemporary Appraisal of the Milan Criteria. Transplantation 2018;102:648-55.

25. Renne SL, Woo HY, Allegra S, et al. Vessels Encapsulating Tumor Clusters (VETC) Is a Powerful Predictor of Aggressive Hepatocellular Carcinoma. Hepatology 2020;71:183-95.

26. Belghiti J, Cortes A, Abdalla EK, et al. Resection prior to liver transplantation for hepatocellular carcinoma. Ann Surg 2003;238:885-92; discussion 892-3.

27. Ferrer-Fàbrega J, Forner A, Liccioni A, et al. Prospective validation of ab initio liver transplantation in hepatocellular carcinoma upon detection of risk factors for recurrence after resection. Hepatology 2016;63:839-49. 